Kalpa Publications in Engineering
Volume 3, 2020, Pages 118-122
Proceedings of International Sym-
posium on Applied Science 2019

\title{
Effects of temperatures on pressure-induced structural changes in amorphous Si: A molecular- dynamics study
}

\author{
Renji Mukuno $^{1 *}$ and Manabu Ishimaru ${ }^{1 \dagger}$ \\ ${ }^{1}$ Kyushu Institute of Technology, Fukuoka, Japan \\ Mukuno.renji711@mail.kyutech.jp, ishimaru@post.matsc.kyutech.ac.jp
}

\begin{abstract}
The structural changes of amorphous silicon (a-Si) under compressive pressure were examined by molecular-dynamics simulations using the Tersoff interatomic potential. aSi prepared by melt-quenching methods was pressurized up to $30 \mathrm{GPa}$ under different temperatures $(300 \mathrm{~K}$ and $500 \mathrm{~K})$. The density of a-Si increased from 2.26 to $3.24 \mathrm{~g} / \mathrm{cm}^{3}$ with pressure, suggesting the occurrence of the low-density to high-density amorphous phase transformation. This phase transformation occurred at the lower pressure with increasing the temperature, because the activation barrier for amorphous-to-amorphous phase transformation could be exceeded by thermal energy. The coordination number increased with pressure and time, and it was saturated at different values depending on the pressure. This suggested the existence of different metastable atomic configurations in a-Si. Atomic pair-distribution functions and bond-angle distribution functions suggested that the short-range ordered structure of high-density a-Si is similar to the structure of the high-pressure phase of crystalline $\mathrm{Si}$ ( $\beta$-tin and Imma structures).
\end{abstract}

\section{Introduction}

A silicon ( $\mathrm{Si}$ ) is a group-IV semiconductor, and it is widely used for various electric devices, because of its excellent physical properties. Previous studies reported that a phase transformation in $\mathrm{Si}$ occurs by applying pressure. Crystalline $\mathrm{Si}$ has a diamond structure at ambient temperature and pressure, while it transforms to the $\beta$-tin structure under high pressure (Durandurdu, 2008). Amorphous $\mathrm{Si}(\mathrm{a}-\mathrm{Si})$ has a four-fold coordinated network at normal pressure. It has been reported that the coordination number increases up to 6 with increasing compressive pressure. This amorphous-toamorphous phase transformation is called "polyamorphism" (Poole, Grande, Angell, \& McMillan, 1997) (Deb, Wilding, Somayazule, \& McMillan, 2001) (McMillan, Wilson, Daisenberger, \& Machon,

\footnotetext{
* Masterminded EasyChair and created the first stable version of this document

${ }^{\dagger}$ Created the first draft of this document
} 
2005) (Daisenberger, et al., 2007) (Durandurdu \& Drabold, 2001) (Morishita, 2004) (Morishita, 2009). Since a crystal has the periodicity and symmetry in its structure, the phase transformation can be understood from a theoretical viewpoint of "group theory". On the other hand, the details of polyamorphism remain an open question, because of a lack of the symmetry and long-range order in the amorphous networks.

A molecular-dynamics (MD) simulation is a powerful technique to investigate the static and dynamic properties of materials at the atomic scale. For MD simulation, the selection of the interatomic potential is very important. Among several empirical interatomic potentials for $\mathrm{Si}$, it has been confirmed that the Tersoff potential can reproduce the amorphous structures of Si (Ishimaru, Munetoh, \& Motooka, 1997) (Kohno \& Ishimaru, 2018). We have recently found that this potential is useful for describing the pressure-induced structural changes of a-Si (Mukuno \& Ishimaru, 2019). The purpose of the present study is to investigate the effect of temperature on the pressure-induced phase transformation of a-Si.

\section{Simulation Procedures}

a-Si was prepared by melt-quenching methods. $4096 \mathrm{Si}$ atoms were initially arranged on a diamond structure with a size of $8 a_{0} \times 8 a_{0} \times 8 a_{0}$, where $a_{0}$ is an initial lattice parameter (5.43 $\AA$ ). This $\mathrm{MD}$ cell was heated at $5000 \mathrm{~K}$ for $100 \mathrm{ps}$ and liquid $\mathrm{Si}$ was prepared. This liquid was quenched from $5000 \mathrm{~K}$ to $300 \mathrm{~K}$ with a cooling rate of $10^{12} \mathrm{~K} / \mathrm{s}$. It was confirmed that the resultant atomic configurations reproduce the amorphous structures of $\mathrm{Si}$. To examine the temperature dependence of the pressure-induced phase transformation, the compressive pressure was gradually increased at 300 $\mathrm{K}$ (room temperature) and $500 \mathrm{~K}$. To obtain detailed structural information, the MD cell was kept at a certain pressure ranging from $10 \mathrm{GPa}$ to $20 \mathrm{GPa}$ (every $2.5 \mathrm{GPa}$ ) for $200 \mathrm{~ns}$. The atomic configuration of a-Si was evaluated by using atomic pair-distribution functions, bond-angle distribution functions, and coordination numbers. In the present study, the interactions between atoms were calculated using the empirical interatomic potential proposed by Tersoff (Tersoff, 1988).

\section{Results and Discussion}

To investigate the pressure-induced polymorphism of $\mathrm{Si}$, the as-quenched a-Si was gradually compressed up to $30 \mathrm{GPa}$. Figure 1 shows the temperature dependence of density change under compressive pressure with a pressure rate of $50 \mathrm{~Pa} / \mathrm{fs}$. The density of the as-quench MD cell before pressurization is $2.26 \mathrm{~g} / \mathrm{cm}^{3}$, which is $\sim 3.0 \%$ lower than that of crystalline $\mathrm{Si}\left(2.33 \mathrm{~g} / \mathrm{cm}^{3}\right)$. The density of the MD cell increases linearly with pressurization. On the other hand, the slope changes at a certain pressure which corresponds to the phase transformation pressure. The location of the kink moves toward lower pressure with increasing the temperature: $\sim 20 \mathrm{GPa}$ for $300 \mathrm{~K}$ and $\sim 12.5 \mathrm{GPa}$ for $500 \mathrm{~K}$. It means that the activation barrier for amorphous-to-amorphous phase transformation is exceeded by thermal energy. After completing the phase transformation, the linear increase of the density was again observed. It should be noted that the density becomes the same between $300 \mathrm{~K}$ and $500 \mathrm{~K}$ at $30 \mathrm{GPa}$, thought the phase transformation pressure is different between them. This suggest that the phase transformation path is the same between these temperatures. 


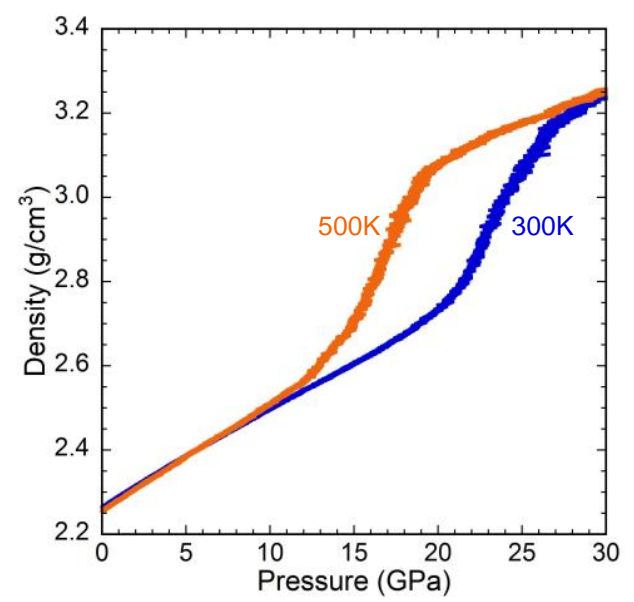

Figure 1: Temperature dependence of density change with pressurization. The MD cell was maintained at $300 \mathrm{~K}$ or $500 \mathrm{~K}$ during the simulations.

To examine the structural change of a-Si, compressive pressures up to $20 \mathrm{GPa}$ were applied to asquenched a-Si under isothermal and isobaric conditions (NPT ensemble). Figure 2 shows the time dependence of the coordination number under compressive pressure. The coordination number of the as-quenched a-Si is $\sim 4$, and the tetrahedral short-range order, so-called low-density a-Si (McMillan, Wilson, Daisenberger, \& Machon, 2005), is maintained up to $10 \mathrm{GPa}$. Above $12.5 \mathrm{GPa}$ the coordination number increases with time. The coordination number increases with pressure, and finally it becomes 5.4 at $20 \mathrm{GPa}$ : high-density a-Si is formed. The coordination numbers are saturated at different values depending on the pressure, suggesting that there are different metastable atomic configurations in a-Si at each pressure.

Figure 3(a) shows the atomic pair-distribution functions, $\mathrm{g}(r)$, as a function of compressive pressure. The $\mathrm{g}(r)$ is defined as the probability of finding another atom at a distance between $r$ and $r+\Delta r$ from a specific atom. The $\mathrm{g}(r)$ converges to the unity at the large $r$-region, indicating the lack of long-range order. In the as-quench a-Si, the first and second peaks are located at $2.36 \AA$ and $3.86 \AA$, respectively, in agree with those obtained experimentally (Moss \& Graczyk, 1969). They move to the shorter distance side at $10 \mathrm{GPa}$. The first peak gradually shifts to the longer distance side for $\geq 12.5$ $\mathrm{GPa}$. This is because the bonding energy per atom decreased with increasing the coordination number. The second peak splits to two peaks of $3.14 \AA$ and $3.72 \AA$ at $15 \mathrm{GPa}$, and the former becomes prominent at $20 \mathrm{GPa}$.

Figure 3(b) shows the bond-angle distribution functions, $\mathrm{g}(\theta)$, as a function of compressive pressure. The $\mathrm{g}(\theta)$ is defined as the probability of bond angles composed of atoms within the first nearest neighbor distance from an arbitrary atom. A peak exists around the tetrahedral angle of $109.5^{\circ}$ in as-quenched a-Si, indicating that the amorphous network consists of the tetrahedral short-range order unit. Upon pressurization, the main peak shifts from the tetrahedral angle to $80^{\circ}$. A $60^{\circ}$ peak also increases gradually. The $\beta$-tin and Imma structures, which are high-pressure phases of crystalline Si (Soma, Iwanami, \& Matsuo, 1982) (McMahon \& Nelmes, 1993), possess the similar bond angles, suggesting that pressure-induced a-Si possesses short-range order similar to the $\beta$-tin and Imma structures. 


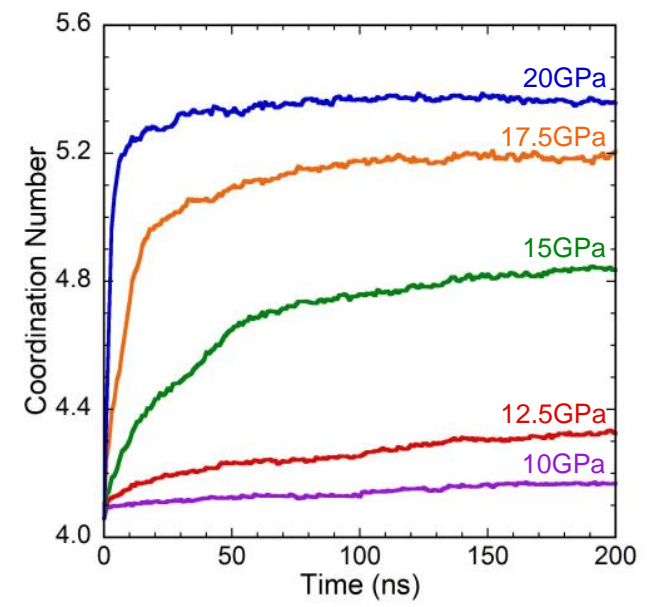

Figure 2: Time evolution of the coordination number under different pressure.
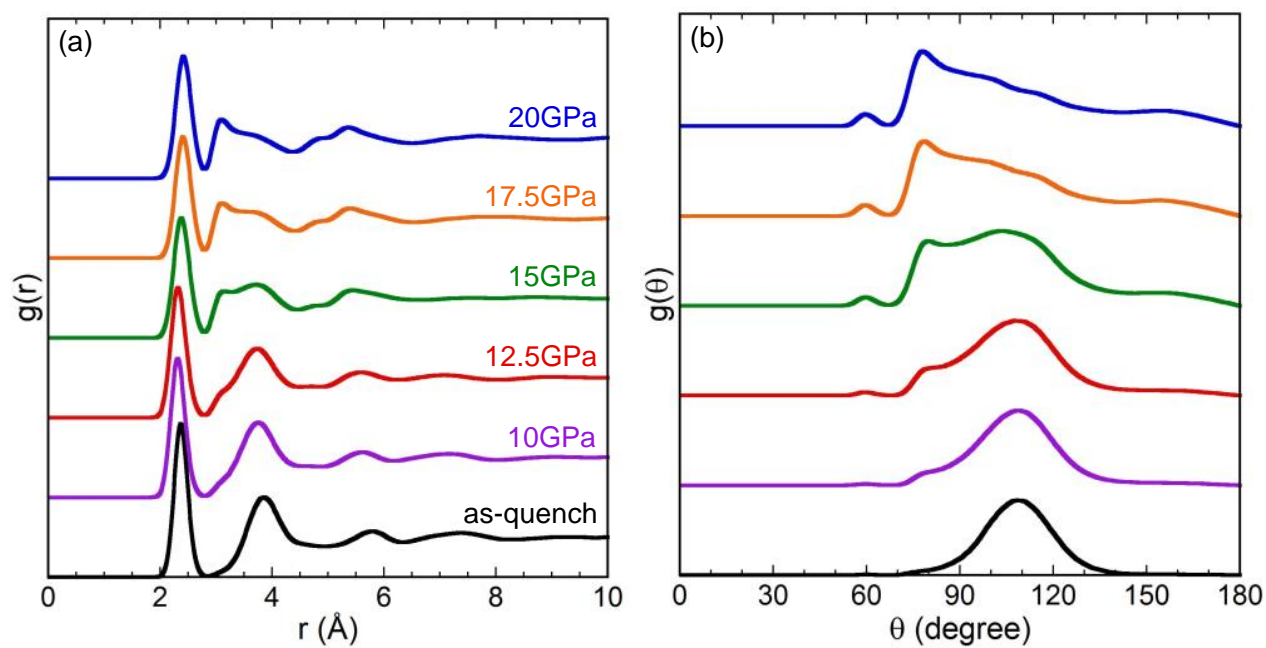

Figure 3: (a) Atomic pair-distribution functions and (b) bond-angle distribution functions of atomic configurations obtained by different pressure.

\section{Summary}

The pressure-induced phase transformation of a-Si was examined by MD simulation using the Tersoff interatomic potential. The phase transformation pressure decreased with increasing the temperature, but the phase transformation path did not change. The coordination number increased with pressure and time, and it was saturated at different values depending on the pressure, suggesting the existence of different metastable states. The atomic pair-distribution function as a function of pressure revealed that the first peak gradually moves to the longer distance side, whereas the second peak sifted to the shorter distance side. The main peak of the bond-angle distribution function changed from $109.5^{\circ}$ (the tetrahedral angle) to $80^{\circ}$. In addition, a new peak appeared at $\sim 60^{\circ}$. After 
the phase transformation, it was suggested that a structure similar to the high-pressure phases of crystalline $\mathrm{Si}$, so-called $\beta$-tin and Imma structure, were formed.

\section{References}

Daisenberger, D., Wollson, M., McMillan, P. F., Cabrera, R. Q., Wilding, M. C., \& Manhon, D. (2007). High-pressure x-ray scattering and computer simulation studies of density-induced polyamorphism in silicon. Physical Review B.

Deb, S. K., Wilding, M., Somayazule, M., \& McMillan, P. F. (2001). Pressure-induced amorphization and an amorphous-amorphous transition in densified porous silicon. nature.

Durandurdu, M. (2008). Diamond to $\beta$-Sn phase transition of silicon under. Journal of Physics: Condensed Matter.

Durandurdu, M., \& Drabold, D. A. (2001). Ab initio simulation of first-order amorphous-toamorphous phase transition of silicon. Physical Review B.

Ishimaru, M., Munetoh, S., \& Motooka, T. (1997). Generation of amorphous silicon structures by rapid quenching: A molecular-dynamics study. Physical Review B.

Kohno, K., \& Ishimaru, M. (2018). Molecular-dynamics simulations of solid phase epitaxy in silicon: Effects of system size, simulation time, and ensemble. Japanese Jounal of Applied Physics.

McMahon, M. I., \& Nelmes, R. J. (1993). New high-pressure phase of Si. Physical Review B.

McMillan, P. F., Wilson, M., Daisenberger, D., \& Machon, D. (2005). A density-driven phase transition between semiconducting and metallic polyamorphs of silicon. Nature Materials.

Morishita, T. (2004). High Density Amorphous Form and Polyamorphic Transformations of Silicon. Physical Review Letters.

Morishita, T. (2009). Structural, electronic, and vibrational properties of high-density amorphous silicon: A first-principles molecular-dynamics study. Journal of Chemical Physics.

Moss, S. C., \& Graczyk, J. F. (1969). Evidence of Voids Within the As-Deposited Structure of Glassy Silicon. Physical Review Letters.

Mukuno, R., \& Ishimaru, M. (2019). Application of the Tersoff interatomic potential to pressureinduced polyamorphism of silicon. Japanese Jounal of Applied Physics.

Poole, P. H., Grande, T., Angell, A. C., \& McMillan, P. F. (1997). Polymorphic Phase Transitions in Liquids and Glasses. Science.

Soma, T., Iwanami, H., \& Matsuo, H. (1982). Phase transition under pressure of Si-Ge solid solutions. Solid State Communications.

Tersoff, J. D. (1988). New empirical approach for the structure and energy of covalent systems. Physical Review B. 